\title{
CLOT FORMATION AND METHYLENE BLUE REDUCTION IN MILK BASED MEDIUM FOR IDENTIFICATION OF CANDIDA SPP. AND TRICHOSPORON SPP.
}

\author{
${ }^{1}$ Trainee, Department of Biotechnology, Patna University. \\ ${ }^{2}$ Assistant Professor, Department of Microbiology, AIIMS, Patna. \\ 3Tutor, Department of Microbiology, AIIMS, Patna. \\ ${ }^{4}$ Senior Resident, Department of Microbiology, AIIMS, Patna. \\ 5 Senior Resident, Department of Microbiology, AIIMS, Patna. \\ ${ }^{6}$ Senior Resident, Department of Microbiology, AIIMS, Patna. \\ 7Tutor, Department of Microbiology, AIIMS, Patna. \\ ${ }^{8}$ Tutor, Department of Microbiology, AIIMS, Patna.
}

Afreen ${ }^{1}$, Sayan Bhattacharyya ${ }^{2}$, Ashwini Kumar ${ }^{3}$, Asim Sarfraz ${ }^{4}$, Abhishek Sengupta ${ }^{5}$, Dhirendra Kumar ${ }^{6}$, Rohit Kumar ${ }^{7}$, Shweta Singh ${ }^{8}$

\section{ABSTRACT}

Candidiasis is one of the most important opportunistic fungal infections in man caused by over 20 species of Candida. After the genus Candida, Trichosporon is the most commonly reported agent of disseminated infections caused by yeasts. In the routine microbiology laboratory, yeast species are frequently identified on the basis of culture characteristics, germ tube production, carbohydrate fermentation reactions and chlamydospore formation by Dalmau technique. These conventional methods usually lack specificity and require longer incubation period resulting in delayed identification, which can lead to a delay in empirical therapies of such infections. The present study, thus, evaluated the reliability of methylene blue reduction test for the rapid identification of Candida and Trichosporon species. Results indicated that clot formation in milk could be reliably used for speciation of these yeasts.

\section{KEYWORDS}

Candida, Trichosporon, Methylene Blue Milk Reduction, Clot.

HOW TO CITE THIS ARTICLE: Afreen, Bhattacharyya S, Kumar A, et al. Clot formation and methylene blue reduction in milk based medium for identification of candida spp. and trichosporon spp. J. Evolution Med. Dent. Sci. 2016;5(48):3044-3047, DOI: $10.14260 /$ jemds/2016/709

\section{INTRODUCTION}

The Human microbiota plays an inevitable role in human metabolism and in understanding the pathogenesis and the optimized therapy for many diseases. In our body, populations of microbes such as bacteria and yeasts form part of normal healthy human flora.(1) However, when the host's cellmediated immune defences are altered, as in immunosuppressed states like HIV infection or during steroid or antibacterial therapy or long-term catheterization or haemodialysis, microbial numbers grow beyond their normal ranges and cause infections.(1) One such opportunistic infection is Candidiasis. It is a fungal infection caused by yeasts that belong to the genus Candida. There are over 20 species of Candida yeasts that can cause infection in humans, the most common of which is Candida albicans. Other Candida species that can cause significant superficial and deep-seated infections include C. tropicalis, C. glabrata, C. parapsilosis, C. stellatoidea, C. dubliniensis and C. krusei.(2)

Candida species are the fourth most common reported agent of bloodstream infections among hospitalised patients in the United States. (3) Chakrabarti etal, in a study in Chandigarh, North India, reported quite high rates of Candida bloodstream infection (Candidaemia) in children in 27 Intensive Care Units (ICU) in India.(4) It is, therefore, of utmost importance to

Financial or Other, Competing Interest: None.

Submission 28-04-2016, Peer Review 23-05-2016,

Acceptance 28-05-2016, Published 14-06-2016.

Corresponding Author:

Dr. Sayan Bhattacharyya

Assistant Professor,

Department of Microbiology,

AIIMS, Patna-801507, Bihar, India.

E-mail: sayantheboss@yahoo.co.in

DOI: $10.14260 /$ jemds/2016/709 identify the species of Candida correctly for the successful treatment of the disease. The conventional methods of identifying these yeast species in the clinical microbiology laboratory include either direct microscopic examination using Potassium Hydroxide $(\mathrm{KOH})$ from the sample or laboratory culture using specified media. Identification by culture methods relies on criteria such as colony characteristics and morphology on different media and sugar assimilation or fermentation reactions. Isolates of $\mathrm{C}$. albicans are typically identified by their ability to form germ tubes (Germ Tube Test) or single terminal chlamydospores (In Dalmau Plate Technique) under the appropriate conditions.(5)

The germ tube test, also known as Reynolds-Braude phenomenon, first described in 1960, is still very useful and accurate for rapid presumptive identification of the yeasts.(6) Although quite rapid, the germ tube test has many shortcomings. Over inoculation of the yeasts in serum can inhibit germ tube formation and too short incubation time (Less than two to four hours) can lead to false negative results, as it may be difficult to discern a true germ tube from that of an early pseudohyphal cell. Additionally, specificity can be lacking as C. tropicalis has been reported to rarely form germ tubes.(7) The use of human serum in this test has several disadvantages. For example, the serum has to be fresh or frozen, better pooled; the yeast inoculum has to contain $<10^{7}$ cells /mL; otherwise, the germ tube production is inhibited. In addition, the handling of pooled human serum for germ tube test includes the possible risk of transmission of HIV or Hepatitis B virus and hence to overcome this risk other media or tests can be used; also, different batches of serum may produce variable results. ${ }^{(8)}$

Another accepted approach for yeast identification is the Dalmau Plate Technique, which requires longer (two days or more) incubation of isolates at room temperature on 
specialised agar-based media (e.g. corn meal agar, corn meal agar with Tween 80 , rice extract agar, rice extract agar with Tween 80, etc.) that is observed microscopically for the presence of yeast cells, pseudohyphae and chlamydospores. $(9,10)$ Since both methods above demand microscopic observation that requires considerable skill and experience, there is a need to develop alternate tests that are equally sensitive and specific, but require less technical expertise, are hence easily interpretable and need shorter incubation time.(11)

The present study was, therefore, undertaken to evaluate Methylene Blue Reduction Test (MBRT) and clot formation in milk as alternative tests to previously described conventional methods of yeast identification in routine clinical microbiology practice.

\section{MATERIALS AND METHODS Type of Study}

This was a laboratory-based observational study, which was carried out in the Department of Microbiology of our Institute, a tertiary care teaching hospital cum referral centre in Eastern part of India as a part of summer training cum dissertation.

\section{Duration of Study}

The study was carried out in 3 months starting from February 2016 to April 2016.

\section{METHOD}

\section{Sources of Isolates}

Yeast isolates were selected from the clinically recovered isolates of Candida and Trichosporon species cultured on Sabouraud dextrose agar slants from samples received in the Microbiology Laboratory.

\section{Isolation and Purification}

The clinical isolates of yeast were first examined under microscope by preparing Lactophenol Cotton Blue (LPCB) mounts from Sabouraud dextrose agar slants. The selected yeast isolates were subsequently purified on fresh Sabouraud Dextrose Agar or SDA (Glucose 2 g; Peptone 1 g; Agar 2 g; Deionized water $100 \mathrm{~mL}$ ) slants. The slants were incubated at $370 \mathrm{C}$ for 24 hours. These purified isolates were identified by conventional methods.

\section{CONVENTIONAL METHODS FOR YEAST IDENTIFICATION Germ Tube Test}

The yeast isolates were inoculated into $0.5 \mathrm{~mL}$ of pooled fresh human serum left over after carrying out routine tests in Biochemistry laboratory and incubated in a water bath at $37^{\circ}$ C for 2 hours. After incubation, a drop of suspension (40 microlitre) was placed on a clean glass slide and mounted with a flame-sterilised glass cover slip and examined under microscope (10x and 40x) for germ tube formation. $(12,13)$

\section{High Temperature $\left(4^{\circ} \mathrm{C}\right)$ Tolerance Test}

Germ tube positive isolates were streaked on SDA slants and incubated in a water bath at $45^{\circ} \mathrm{C}$. After 24 hours, growth was observed to facilitate differentiation of C. albicans and C. dubliniensis. Positive growth next day indicated C. albicans, while no growth at this temperature denoted C. dubliniensis.(14)

\section{Morphology on Rice Extract Agar (Dalmau Technique)}

Light inoculum of the yeast isolates was partially streaked into half the thickness of Rice Extract Agar (REA) (Rice, powdered, $0.04 \mathrm{~g}$; Agar agar $1.5 \mathrm{~g}$; Deionized water $100 \mathrm{~mL}$ ) media making 4-5 parallel lines of approximately 2 to $2.5 \mathrm{~cm}$ long and 0.5 to $0.8 \mathrm{~cm}$ apart. A flame sterilized cover slip was placed over it and incubated at $22^{\circ} \mathrm{C}$ for 3 to 5 days. After incubation, the lid of the petri plate was removed and the edge of the cover slip was observed under the microscope (10X and 40X objectives) for typical morphological features like yeast cells, hyphae, pseudohyphae, chlamydospores or arthroconidia along with budding yeast cells for Trichosporon spp. $(13,14,15)$ Urease test was done on Christensen's urea agar slant to identify Trichosporon spp. since it was found to be Urease positive.

\section{Sugar Fermentation}

Nutrient broth with glucose, maltose, sucrose and lactose, each in concentration of $2 \%$ (weight/volume) with phenol red indicator with appropriate colour on cotton plug for differentiation were prepared and sterilised by autoclaving at $110^{\circ} \mathrm{C}$ at $10 \mathrm{lbs} / \mathrm{in}^{2}$ pressure for 10 minutes. One loopful of yeast isolate was inoculated in $2 \mathrm{~mL}$ of the liquid medium in test tubes and incubated at $37{ }^{\circ} \mathrm{C}$ overnight with known strains of Candida spp. as controls. A yellow colouration was indicative of positive fermentation reaction.

\section{Characterization of the selected Isolates for MBRT}

The isolates were characterized by evaluating their methylene blue reduction ability in milk-based medium.

\section{Methylene Blue Reduction Test}

The yeast isolates were inoculated in methylene blue milk liquid medium (Methylene blue solution, 0.04\% $1 \mathrm{~mL}$; fresh whole fat Milk $100 \mathrm{~mL}$, sterilised by autoclaving at $110^{\circ} \mathrm{C}$ at 10 $\mathrm{lbs} / \mathrm{in}^{2}$ pressure for 10 minutes) and incubated at $37^{\circ} \mathrm{C}$ for 24 hours in incubator. The gradual change in the colour of media from blue to white indicated positive reduction; clot formation was also observed.(15) Clotting was observed by inverting the test tube. Positive clot formation was inferred when the suspension of yeast in milk did not fall down on inverting the test tube upside down.

\section{RESULTS}

A total of 35 yeast isolates belonging to different species were recovered from various clinical samples in this period of time (Like blood, urine, pus and sputum). These clinical isolates namely C. albicans, C. dubliniensis, C. tropicalis, C. kefyr, C. glabrata, C. parapsilosis and T. cutaneum were presumptively identified to the species level by using germ tube test, high temperature $\left(45^{\circ} \mathrm{C}\right)$ tolerance test, carbohydrate fermentation test, urease test and by microscopic examination of chlamydospore, pseudohyphae, budding yeasts and arthroconidia production on Rice Extract Agar (REA) medium.

\section{Methylene Blue Reduction Test}

All the isolates of C. dubliniensis, C. tropicalis, C. kefyr, C. parapsilosis, C. glabrata, $\mathrm{T}$. cutaneum and $81.25 \%$ isolates of $\mathrm{C}$. albicans were found to be positive for MBRT test. Clot formation was observed in $37.50 \%$ C. albicans, $50 \% \mathrm{~T}$. cutaneum, $25 \%$ C. parapsilosis, $25 \%$ C. tropicalis and in all the isolates of C. dubliniensis and C. kefyr (Table 1). All tests were done three times with each isolate. 


\begin{tabular}{|c|c|c|c|c|c|}
\hline $\begin{array}{c}\text { Yeast } \\
\text { Species }\end{array}$ & $\begin{array}{c}\text { No. } \\
\text { of } \\
\text { Isolates }\end{array}$ & $\begin{array}{c}\text { MBRT } \\
\text { Test } \\
\text { (No. of } \\
\text { Positive/ } \\
\text { out of } \\
\text { Total) }\end{array}$ & $\begin{array}{c}\text { Clot } \\
\text { formation } \\
\text { (No. of } \\
\text { positive/ } \\
\text { Out of } \\
\text { Total) }\end{array}$ & $\begin{array}{c}\text { \% } \\
\text { Positive } \\
\text { (MBRT } \\
\text { Test) }\end{array}$ & $\begin{array}{c}\text { \% Positive } \\
\text { (Clot } \\
\text { Formation) }\end{array}$ \\
\hline C. albicans & 16 & $13 / 16$ & $6 / 16$ & 81.25 & 37.50 \\
\hline C. dubliniensis & 2 & $2 / 2$ & $2 / 2$ & 100 & 100 \\
\hline C. glabrata & 6 & $6 / 6$ & $0 / 6$ & 100 & 0 \\
\hline C. kefyr & 1 & $1 / 1$ & $1 / 1$ & 100 & 100 \\
\hline C. parapsilosis & 4 & $4 / 4$ & $1 / 4$ & 100 & 25 \\
\hline C. tropicalis & 4 & $4 / 4$ & $1 / 4$ & 100 & 25 \\
\hline T. cutaneum & 2 & $2 / 2$ & $1 / 2$ & 100 & 50 \\
\hline \multicolumn{2}{|r|}{ Table 1: Results of MBRT Test and Clot Formation } \\
\hline \multicolumn{7}{|r|}{} \\
\hline
\end{tabular}

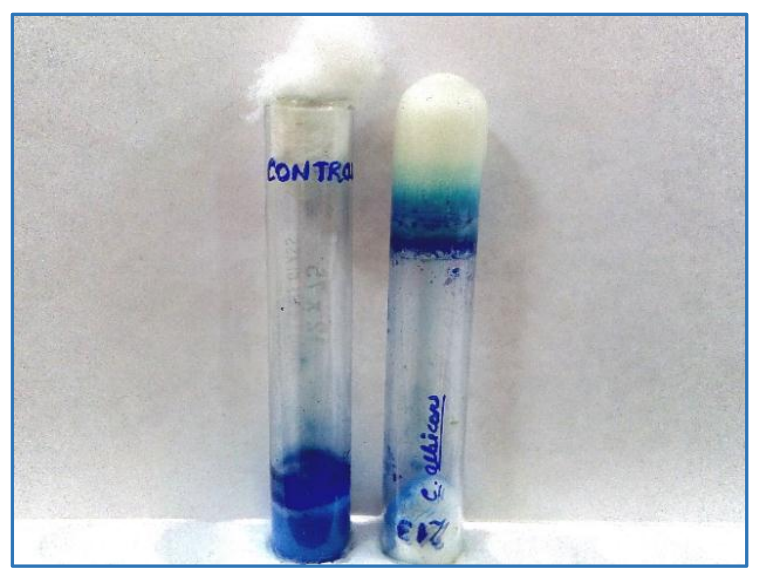

Fig. 1: Clot Formation in C. albicans

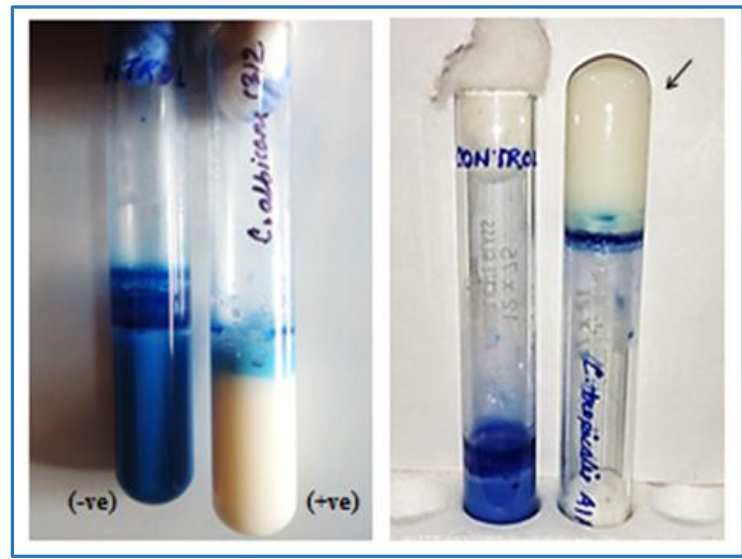

Fig. 2: MBRT and Clot Formation

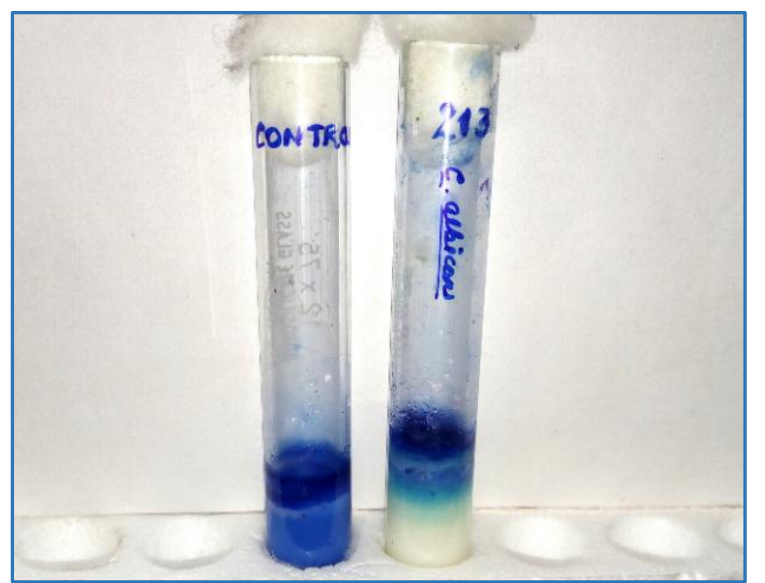

Fig. 3: Methylene Blue Reduction in C. albicans

\section{DISCUSSION}

The conventional methods for the identification of Candida spp., although simple and inexpensive impose some serious limitations on the microbiologist. These methods are known to be time consuming and more often unable to discriminate between the different species (For example germ tube test fails to differentiate between C. albicans and C. dubliniensis) and thus lack specificity. Recognition of yeast morphologies on Dalmau plates requires some experience and expertise as well. Misidentification of yeast species may have some serious clinical implications as regards to treatment.

Newer techniques like real time PCR, matrix assisted laser desorption ionization time of flight mass spectrometry (MALDI-TOF) and multiplex tandem PCR are now being evaluated and employed for the rapid identification of yeast species.(16-19) More recent methods like Vitek-2 system requires at least 15 hours incubation with isolates grown in culture, almost same as with the new tests described here.(20) Moreover, for using Vitek-2, cultures cannot be more than 24 hours old, because then results can be unreliable.(21) CHROM agar can be used for identification, but results can be inferior to conventional methods for Candida albicans identification, and can also lead to wrong identification in some species. (22) Also, such expensive techniques are not commonly available for use in routine and peripheral clinical microbiology laboratories.

The present study, therefore, evaluated two simple and cost effective alternative methods for the identification of different Candida and Trichosporon species. Methylene Blue Reduction Test (MBRT) is routinely done in dairy industry to assess bacteriological quality of milk.(23) This test can be used to rapidly and accurately evaluate yeast vitality. ${ }^{(24)}$ In this study, 32 out of 35 (91.42\%) yeast isolates in total were found to be MBRT positive. Out of these, 6 out of $16(37.50 \%) \mathrm{C}$. albicans, 1 of 4 (25\%) C. parapsilosis, 1 out of 4 (25\%) C. tropicalis and 1 out of $2(50 \%)$ T. cutaneum strains showed positive clot formation. Clot formation was always found to be positive in C. dubliniensis and C. kefyr. In fact, C. kefyr was positive for both MBRT as well as clot formation. No clot formation was observed in C. glabrata (Table 1). C. albicans was not always MBRT positive (negative in about $18 \%$ cases). Clot formation of milk has previously been documented in Cryptococcus spp. and Rhodotorula spp., but not in Candida spp. and Trichosporon spp.(25)

These newer tests are simple and cost effective, that will aid routine yeast identification or can be adjunct to routine techniques leading to accurate and faster clinical diagnosis for instituting early and correct antifungal therapy. Clotting of milk by liberation of proteases has recently been demonstrated in Aspergillus tamarii and Penicillium pinophilum.(26) As far as we know, these tests have not yet been used or assessed for identification of Candida spp. and Trichosporon spp. from clinical samples. MBRT and milk clotting tests should therefore be further assessed and more such newer cheap tests are needed to be developed urgently in coming future. The present study, however, has a limitation as it was carried out with only 35 yeast isolates belonging to 7 species only. So, these alternative tests are needed to be carried out on many other clinically significant yeast species that are not studied in this research and with a larger number of yeast isolates. 


\section{CONCLUSION}

Because of the lack of specificity and delay in obtaining identification by conventional methods, there is a need to evaluate alternative tests and suitable methods for rapid identification of different Candida and Trichosporon species in a routine microbiology laboratory.

\section{ACKNOWLEDGEMENT}

Authors like to acknowledge the overall help and support of Ravi Kumar and Mahesh Kumar, Lab Attendants in the Department.

\section{REFERENCES}

1. Chakravarthi S, Haleagrahara N. A comprehensive review of the occurrence and management of systemic candidiasis as an opportunistic infection. Microbiol J 2011;1(1):1-7. doi: 10.3923/mj.2011.1.7.

2. Kwon-Chung KJ, Bennett JE, Cann C. Candidiasis moniliasis, thrush, candida paronychia, candida endocarditis, bronchomycosis, mycotic vulvovaginitis, candiosis. Med mycol 1992;280-336.

3. Candidiasis (pdf). Candidiasis center of disease control and prevention 2015. www.cdc.gov/fungal/infections/

4. Chakrabarti A, Sood P, Rudramurthy SM, et al. Incidence, characteristics and outcome of ICU-acquired candidemia in India. Intensive Care Med 2015;41(2):285-95. doi: 10.1007/s00134-014-3603-2.

5. Deorukhkar S, Saini S. Laboratory approach for diagnosis of candidiasis through ages. Int J Curr Microbiol Appl Sci 2014;3(1):206-218.

6. Taschdjian CL, Burchall JJ, Kozinn PJ. Rapid identification of candida albicans by filamentation on serum and serum substitutes. Am J Dis Child 1960;99:212-5.

7. Tierno PM, Milstoc M. Germ tube-positive candida tropicalis. Am J Clin Pathol 1977;68(2):294-5.

8. Mattei AS, Alves SH, Severo CB, et al. Use of mueller hinton broth and agar in the germ tube test. Revista do Instituto de Medicina Tropical de São Paulo 2014;56(6):483-5. doi:10.1590/S0036-46652014000600005.

9. Vander Walt JP, Yarrow D. Methods for the isolation, maintenance, classification and identification of yeasts. In: Kreger-van Rij NJW (ed). The yeasts: a taxonomic study. Amsterdam: Elsevier Science Publishers BV 1984;3rd ed:45-104.

10. Barnett JA, Payne RW, Yarrow D. Yeasts: characteristics and identification. Cambridge: Cambridge university press, 2000; $3^{\text {rd }}$ ed.

11. Pincus DH, Orenga S, Chatellier S. Yeast identification past, present, and future methods. Med Mycol 2007;45(2):97-121. doi: 10.1080/13693780601059936.

12. Standard Operating Procedures (PDF). National antifungal resistance surveillance.

www.icme.nic.in/publications 2015.
13. Raju SB, Rajappa S. Isolation and identification of candida from the oral cavity. ISRN Dentistry Article ID 487921, 2011;p 7. doi:10.5402/2011/487921.

14. Pinjon E, Sullivan D, Sulkin I, et al. Simple, inexpensive, reliable method for differentiation of candida dubliniensis from candida albicans. J Clin Microbiol 1998;36(7):2093-5.

15. Atherton HV, Newlander JA. Chemistry and testing of dairy products. AVI, Westport, CT 1977; $4^{\text {th }}$ ed.

16. Khan Z, Mustafa AS, Alam FF. Realtime lightcycler polymerase chain reaction and melting temperature analysis for identification of clinically important candida spp. J Microbiol Immunol Infect 2009;42(4):290-5.

17. Lau A, Halliday C, Chen SC, et al. Comparison of whole blood, serum, and plasma for early detection of candidemia by multiplex tandem PCR. J Clin Microbiol 2010;48(3):811-6.

18. Marklein G, Josten M, Klanke U, et al. Matrix assisted laser desorption ionization time of flight mass spectrometry for fast and reliable identification of clinical yeast isolates. J Clin Microbiol 2009;47(9):2912-7.

19. VanVeen SQ, Claas EC, Kuijper EJ. Highthroughput identification of bacteria and yeast by matrixassisted laser desorption ionization time of flight mass spectrometry in conventional medical microbiology laboratories. J Clin Microbiol 2010;48(3):900-7.

20. Graf B, Adam T, Zill E, et al. Evaluation of the VITEK 2 system for rapid identification of yeasts and yeast-like organisms. J Clin Microbiol 2000;38(5):1782-5.

21. Cassonet C, Eldere JV, Vaneechoutte M, et al. Comparison of VITEK 2 with ITS2- fragment length polymorphism analysis for identification of yeast species. J Clin Microbiol 2004;42(5):2209-11.

22. Devi LS, Maheshwari M. Speciation of candida species isolated from clinical specimens by using chrom agar and conventional methods. Int J Sci Res Pub 2014;4(3):1-5.

23. Worku T, Negera E, Nurfeta A, et al. Microbiological quality and safety of raw milk collected from borana pastoral community, oromia regional state. African Journal of Food Science and Technology 2012;3(9):213222.

24. Li W, Guo Z, Zhang L, et al. A novel and rapid method for yeast vitality evaluation based on the methylene blue dye reduction test. J ASBC 2011;69(1):44-9.

25. Federici F. A note on milk clotting ability in the yeast genera cryptococcus and rhodotorula. J Appl Microbiol 1982;52(2):293-6.

26. Benlounissi SA, Mechakra-Maza A, Blum LJ, et al. Identification and characterisation of milk-clotting proteases produced by two species of mold. African J Biotechnol 2014;13(11):1275-80. 